\section{Adhesives Age}

1) U.C. Jindal: Adhesives and stress distribution of a plate with reinforced hole, 26, 8 (1983), 25. *radial stress, circumferential stress, shear stress, Airy's stress function, photoelastic analysis.

\section{AIAA Journal}

1) L. Delneste \& B. Perez: An inelastic finite element model of 4D carbon-carbon composites, 21, 8 (1983), 1143. * *solid rocket nozzle, elastoplastic finite element model, orientation, four directions.

2) K.A. Lincoln: Experimental determination of vapor species from laser-ablated carbon phenolic composites, 21, 8 (1983), 1204. *heat-shiel material, vehicle entry, laser irradiation, mass spectrometry, comparison with theory.

3) R.L. Ramkumar \& P.C. Chen: Low-velocity impact response of laminated plates, 21, 10 (1983), 1448. *transverse shear deformation, contact problem, initial failure, back surface failure, delamination, analysis.

\section{Astronautics and Aeronautics}

1) B. Frisch: Composite structure cheaper than aluminum, 21, 4 (1983), 10. *graphite/epoxy, cylindrical shell, sandwich, weight parameter, price.

2) V. Wigotsky: First design details of the all-composite lear fan, 21, 5 (1983), 30. *lear fan 2100, graphite/epoxy, Kevlar/epoxy, fabric, adhesive bonding.

3) B. Frisch: Composites and hard knocks of space, 21, 5 (1983), 33. *large spacecraft, hard vacuum, thermal cycling, thermal expansion.

4) R.C. Burk: Standard failure criteria needed for advanced composites, 21, 6 (1983), 58. *AIAA survey, failure criteria, summary.

5) J.F. Schier \& R.J. Juergens: Design impact of composites in fighter aircraft, Part 1; They force a fresh look at the design process, 21, 9 (1983), 44. *boron/epoxy, carbon/epoxy, fluidity to design, F-15, F/A-18, AV-8B.

\section{Composites}

1) D. Valentin, Ph. Bonniau \& A.R. Bunsell: Failure mechanism discrimination in carbon fibre-reinforced epoxy composites, 14, 4 (1983), 345. *composite materials, acoustic testing, amplitude analysis, failure mechanism, unidirectional composites, crossplied composites.

2) F.E. Devine: Polyester moulding materials in automotive underbonnet environments, 14, 4 (1983), 353. *composite materials, environmental testing, inlet manifolds, polyester resins.

3) C. Ferrero, G. Genta \& C. Marinari: Experimental strain measurements on bare filament flywheels, 14, 4 (1983), 359. *composite materials, flywheels, strain measurement, stress analysis, spin tests, mathematical models.

4) P.W.M. Peters: On the increasing fracture toughness at increasing notch length of $0 / 90$ and $0 / \pm 45 / 0$ graphite/epoxy laminates, 14, 4 (1983), 365. *composite materials, crak-opening displacement tests, notch length, fracture toughness, damage zone size.

5) A. Koplev, Aa. Lystrup \& T. Vorm: The cutting process, chips, and cutting forces in machining GFRP, 14, 4 (1983), 371. *composite materials, machining, cutting techniques, cutting mechanism, macrochips.

6) H.M. Hinkel: Fabrication of a composite recording disc substrate, 14, 4 (1983), 377. *composite materials, magnetic discs, mechanical testing, glass fibres, carbon fibres, epoxy resins, steel foils.

7) S.K. Malhotra \& B.J.C. Babu: A study on GRP skin/foam core sandwich structures, 14, 4 (1983), 383. *sandwich materials, weight control, design calculations.

8) A. Apicella, C. Migliaresi, L. Nicolais, L. Iaccarino \& S. Roccotelli: The water ageing of unsaturated polyester-based composites: influence of resin chemical structure, 14, 4 (1983), 387. *composite materials, water-absorption tests, polyester resins, hydrolytic stability.

\section{Experimental Mechanics}

1) H. Chai, W.G. Knauss \& C.D. Babcock: Observation of damage growth in compressively loaded laminates, 23, 9 (1983), 329. *phenomenological aspects, panel, failure, in-plane loading, transverse impact, real-time recording, failure-propagation, buckling, delamination.

\section{Fibre Science and Technology}

1) P.S. Theocaris: Dynamic behavior of composites studied by caustics, 19, 3 (1983), 157. *dynamic caustics, fracture behavior, interface, stress intensity factor.

2) B.L. Dhoopar \& P.K. Sinha: Plane stress analysis of cylindrically orthotropic composites, Part 1. A cylindrically reinforced disc subjected to uniform radial pressure, 19, 3 (1983), 179. *cylindrically reinforced composite, modified model, numerical result. 
3) B.L. Dhoopar \& P.K. Sinha: Plane stress analysis of cylindrically orthotropic composites, Part 2. A cylindrically reinforced disc under diametral compression, 19, 3 (1983), 195. *diametral compression, approximate analysis, photoorthotropic elasticity.

4) G.C. Habeger \& W.J. Whitsitt: A mathematical model of compressive strength in paperboard, 19, 3 (1983), 215. *edgewise compression, experiment. empirical relationship, mathematical model.

\section{Forest Products Journal}

1) M.A. Dean \& A.J. Kass: Predicting the bending strength of ladder rail stock, 33, 9 (1983), 9. *multiple variables, safety index, reliability, coefficient of variation.

2) F.K. Bechtel: Proof test load value determination for maximum economic turn, 33, 10 (1983), 30. *failure cumulative distribution function, Weibull form, Gaussian form.

\section{International Journal of Numerical Methods in Engineering}

1) P.J. Sunder, G.V. Ramakrishnan \& S. Sengupta: Finite element analysis of 3-ply laminated conical shell for flutter, 19, 8 (1983), 1183. *aerodynamic pressure, natural frequency, flutter boundary, core thickness.

\section{International Journal of Solids and Structures}

1) J. Aboudi: The effective moduli of short-fiber composites, 19, 8 (1983), 693. *continuum theory, periodic array, rectangular parallelpiped elastic fiber, elastic matrix, periodically bilaminated, particulate.

2) A.I. Beltzer, C.W. Bert \& A.G. Striz: On wave propagation in random particulate composites, 19, 9 (1983), 785. *viscoelastic, scattering effect, viscoelastic loss, Kramers-Kronig relation, attenuation, dispersion.

3) D.R.S. Talbot \& J.R. Wills: Variational estimates for dispersion and attenuation of waves in random composites-III, 19, 9 (1983), 793. *two-dimensional, transverse propagation to fibers, materials reinforced by aligned elliptic cylinder, distribution function, Green's function.

4) S.S. Wang \& F.G. Yuan: A singular hybrid finite element analysis of boundary-layer stresses in composite laminates, 19, 9 (1983), 825. *singular composite-edge element, Pipes-Pagano problem, eigenfunction truncation, size of element, aspect ratio, displacement-based elements.

5) S.K. Datta \& H.M. Ledbetter: Elastic constants of fiber-reinforced boron-aluminum: observation and theory, 19, 10 (1983), 885. *laminated, uniaxial, square-array, hexagonal-array, randomly distributed fiber, anisotropy of boron fiber, transverse-isotropic model.

\section{International Polymer Science and Technology}

1) P. Wiedmann: New method for testing textilereinforced elastomer membranes, 10, 1 (1983), T/24. *tensile test piece (DIN53504), Henker's equipment. 2) E.A. Dzyura, A.V. Kuz'min, L.G. Klimenko \& V.N. Belkovskii: Service life of tractor tyres with a carcass made from a rubber-fiber composite, 10, 4 (1983), T/1. *tyre production, high M-M rubber, tractor tyre.

3) E.A. Bozhevol'naya, A.A. Veshchev \& A.M. Trofimov: Method of increasing structural uniformity in the shaped carcasses of radial tyres, 10, 5 (1983), T/42. *shaping operation, structural uniformity, 165SR 13 .

4) Yu.V. Glukhova, T.V. Kiseleva \& G.S. Klitenik: Method of assessment of the fatigue life of rubbermetal shear mountings, 10, 6 (1983), T/19. *fatigue life, twist angle, temperature.

5) S.D. Plisadov \& V.G. Tsyss: Relationship between the safety factor and reliability of rubber/cord diaphragms, 10, 6 (1983), T/31. *rubber/cord diaphragm, safety factor, failure.

6) G.M. Bartenev \& I.L. Pushkina: Influence of the type of textile base and rubber coating on the diffusion and strength properties of rubber-fabric materials, 10, 6 (1983), T/42. *air permeability, tensile strength, glass transition temperature.

7) V.A. Ishchenko \& L.F. Nikitina: Calculation of the temperature fields of pneumatic tyres, taking into account their state of stress deformation, 10, 6 (1983), $\mathrm{T} / 87$. *tyre design, temperature condition, heat build-up, stress-deformation characteristic.

\section{Journal of Aircraft}

1) J.M. Snead \& A.N. Palazotto: Moisture and temperature effects on the instability of cylindrical composite panels, 20, 9 (1983), 777. *instability, graphite/epoxy, moisture condition, degradation of transverse modulus, finite element analysis.

\section{Journal of Composite Materials}

1) T. Ishikawa \& T.-W. Ghou: In-plane thermal expansion and thermal bending coefficients of fabric composites, 17, 2 (1983), 92. *mosaic model, fiber undulation model, bridging model, plain weave composites, satin weave composites, cross-ply laminates.

2) R. Richards-Frandsen \& Y. Naerheim: Fracture morphology of graphite/epoxy composites, 17, 2 (1983), 105. *graphite/epoxy composites, failure surfaces, three-point bending fatigue, fatigue lifetime. 
3) S.S. Wang \& E.S.-M. Chim: Fatigue damage and degradation in random short-fiber composite, 17, 2 (1983), 114. *random short-fiber SMC composites, fatigue damage, cyclic history.

4) A.C. Loos \& G.S. Springer: Curing of epoxy matrix composites, 17, 2 (1983), 135. *thermosetting resin matrix prepreg materials, thermo-chemical model, resin flow model, void model, stress model.

5) O.A. Bauchau: Optimal design of high speed rotating graphite/epoxy shafts, 17, 2 (1983), 170. *high speed rotating shaft, graphite/epoxy materials, bending stresses, thickness.

6) R.P. Khetan \& D.C. Chang: Surface damage of sheet molding compound panels subject to a point impact loading, 17, 2 (1983), 182. *chopped-glassfiber-reinforced sheet molding compounds, surface damage area, impact damage, SMC panels.

7) Z. Chi \& T.-W. Chou: An experimental study of the effect of prestressed loose carbon strands on composite strength, 17, 3 (1983), 196. *composite strength, loose bundles, gauge length, carbon fiber.

8) S.S. Wang: Fracture mechanics for delamination problems in composite materials, 17, 3 (1983), 210. *composite materials, crack-tip stress, mixed-mode stress, ply thickness, delamination crak.

9) P.J. Fritz \& R.A. Queeney: Interfacial matrix stresses in composites due to misfitting cylindrical constituents, 17, 3 (1983), 224. *composite materials, matrix stress, misfitting cylinders, three-inclusion composite model.

10) A. Hoenig: Thermal conductivities of a cracked solid, 17, 3 (1983), 231. *analysis, dry cracks, filled cracks, thermal conductivity.

11) L. Carlsson: Interlaminar stresses at a hole in a composite member subjected to in-plane loading, 17, 3 (1983), 238. *28-ply graphite/epoxy composite, in-plane loading, finite element method, $6 \mathrm{~mm}$ circular hole.

12) S.S. Wang, E.S.-M. Chim \& N.M. Zahlan: Fatigue crack propagation in random short-fiber SMG composite, 17, 3 (1983), 250. *random shortfiber reinforced SMC composite, fatigue crack growth, single-edge-notched specimen.

13) G.L. Farley: Energy absorption of composite materials, 17, 3 (1983), 267. *energy absorption, graphite/epoxy, Kevlar/epoxy, glass/epoxy, composite tube, aluminum tube.

\section{Journal of Spacecraft and Rockets}

1) N.A. Mumford, P.C. Hopkins \& B.A. Lloyd: Matrix/fiber interface effects on Kevlar 49R pressure vessel performance, 20, 4 (1983), 399. *filament winding, rocket motor case, matrix resin system, fiber prefinish.

\section{Journal of the Mechanics and Physics of Solids}

1) A.J. Belfield, T.G. Rogers \& A.J.M. Spencer: Stresses in elastic plates reinforced by fibres lying in concentric circles, 31, 1 (1983), 25. *transversely isotropic, annulus, inextensible solution, boundary layer theory, circular hole with normal traction.

2) D.W. Kum, T. Oyama, J. Wadsmorth \& O.D. Sherby: The impact properties of laminated composites containing ultrahigh carbon (UHG) steels, 31, 2 (1983), 173. *UHC steel/mild steel, UHC/ UHC laminate, roll bonding procedure, Charpy impact test, 25 to $-196^{\circ} \mathrm{C}$, notch blunting.

3) R. Hill: Interfacial operators in the mechanics of composite media, 31, 4 (1983), 347. *stress discontinuities, splitting second-rank tensor, elastoplastic composite, review, new standpoint.

\section{NASA Contractor Report}

1) J.H. Hemann \& G.Y. Baaklini: The effect of stress on ultrasonic pulses in fiber reinforced composites, GR-3724 (1983). *ultrasonics, attenuation, flexural waves, lamb waves, stress/strain, fiber composites.

\section{NASA Technical Paper}

1) W.S. Johnson, G.A. Bigelow \& Y.A. Bahei-El-Din: Experimental and analytical investigation of the fracture processes of bolon/aluminum laminates containing notches, TP-2187 (1983). *metal matrix composites, fracture, radiography, elastic-plastic finiteelement analysis, fiber failure, constituent material model.

\section{Rubber Chemistry and Technology}

1) H.P. Schlanger: A one-dimensional numerical model of heat transfer in the process of tire vulcanization, 56, 2 (1983), 304. *tire vulcanization, heat transfer.

2) S.K. Clark: Theory of the elastic net applied to cord-rubber composites, 56, 2 (1983), 372. *cordrubber composite, Halpin-Tsai Eq., Gough-Tangorra Eq., Akasaka-Hirano Eq.

3) G.R. Hamed \& T. Donatelli: Effect of accelerator type on brass-rubber adhesion, 56, 2 (1983), 450. *brass-rubber adhesion, mixing, cure ratio, adhesion test.

4) D.K. Setua \& S.K. De: Short silk fiber reinforced natural rubber composites, 56, 4 (1983), 808. *natural rubber, short fiber, silk fiber.

\section{Rubber World}

1) S.K. Moghe: Short fiber reinforcement of elastomers (featuring a mathematical model), 187, 5 
(1983), 16. *short fiber, mathematical model, time dependent behavior.

\section{Wood and Fiber Science}

1) R.Y. Itani: Influence of sheathing gaps on wood floor systems, 15, 3 (1983), 190. * composite structure, load intensity, glucline stiffness, slip modulus, midspan deflection.

2) R.A. Johnson \& W.L. Galligan: Estimating the concomitance of lumber strength properties, 15, 3
(1983), 235. *proofload. correlation, cofunctioning, covariance, conditional likelihood.

3) C.K. Cheung \& H.C. Sorensen: Effect of axial loads on radial stress in curved beams, 15, 3 (1983), 263. *stress function, equation of equilibrium. compatibility equation, Wilson's equation, geometrical parameter, tangential stress, shear stress.

紙面の都合上，キーワードをもって概要に代えた．

*はキーワードを示す.

(情報委員会)

\section{日本複合材料学会誌（邦文誌）への論文投稿のすすめ}

日本複合材料学会誌 (邦文誌) は年 4 回の刊行で, 各号に $3 \sim 4$ 編の邦文論文（研究論文, 研究ノート) の揭 載を予定しております．会員各位の積極的なご投稿をおすすめいたします.

缃投稿資格 本会会員に限りますが，会員外の共同 研究者を含むことはさしつかえありません．

\section{约原稿の種類および制限頁数}

研究論文: 刷上り 6 ページ以内

研究ノート：刷上り 2 ページ以内

技術資料：刷上り 6 ページ以内

织投稿の申込み任意の用紙に著者名, 論文題目, 論文種目, 論文要旨（400 字以内）および連絡先 等を記入のうえ下記宛郵送くだい。

于113 東京都文京区湯島 2-30-9

学会誌刊行センター内

「日本複合材料学会誌」編集委員会 (TEL. 03-815-0499)
折返し，学会所定の原稿用紙（有料）を㮸りい たします。論文作成の要領は原稿用紙の表紙裏面に 印刷してありますのでご参照くたさい.

\section{统揭載 料 無料}

\section{踏刷料金}

\begin{tabular}{crrrr} 
& 50 & 100 & 150 & \multicolumn{1}{c}{200 部 } \\
\hline $1 \sim 2$ 頁 & 12,000 & 16,000 & 20,000 & 24,000 円 \\
$3 \sim 4$ & 18,000 & 22,000 & 26,000 & 30,000 \\
$5 \sim 6$ & 24,000 & 28,000 & 32,000 & 36,000 \\
7 & 40,000 & 45,000 & 50,000 & 55,000 \\
8 & 50,000 & 55,000 & 60,000 & 65,000 \\
\hline
\end{tabular}

(全表紙付, 最低 50 部購入) 